\title{
ABCA1 and $\mathrm{HDL}_{3}$ are Required to Modulate Smooth Muscle Cells Phenotypic Switch
}

after Cholesterol Loading

S. Castiglioni, M. Monti, A. Vettore, L. Arnaboldi, M. Canavesi, A. Corsini, S. Bellosta Department of Pharmacological and Biomolecular Sciences, Università degli Studi di Milano, Milan, Italy.

Aim. Arterial smooth muscle cells (SMCs) may accumulate cholesterol and modify their phenotypic behavior becoming foam cells. We aimed to characterize the role of $\mathrm{HDL}_{3}$ and the ATP binding cassette transporter ABCA1 in this process.

Methods. We evaluated the cholesterol-induced phenotypic changes in SMCs isolated from wild type (WT) and ABCA1 knock out (KO) mice and how $\mathrm{HDL}_{3}$ affects these changes.

Results. Cholesterol loading downregulates the expression of ACTA2 (SMC-marker), and increases the expression of Mac-2, SRB1, ABCG1 and ABCA1 (macrophage-related genes). $\mathrm{HDL}_{3}$ normalizes ACTA2 expression and reduces the expression of macrophagerelated genes in WT cells. Interestingly, the effect of $\mathrm{HDL}_{3}$ is completely lost in $\mathrm{ABCA} 1 \mathrm{KO}$ cells. Concordantly, $A B C A 1$ knock-down by siRNA completely abolishes the rescue effect by $\mathrm{HDL}_{3}$ in WT SMC. The presence of $\mathrm{HDL}_{3}$ does not differently affect cholesterol accumulation in WT or ABCA1 KO cells and stimulates phospholipids removal only in WT cells. Cholesterol loading reduces the expression of myocardin, the key SMC transcriptional coactivator $(-55 \%, p<0.01$ vs control) in both cell types, while increases the expression of KLF4 (a transcriptional factor which represses the expression of myocardin) in WT cells $\left(+240 \%, \mathrm{p}<0.01\right.$ vs control). $\mathrm{HDL}_{3}$ normalizes myocardin and KLF4 levels in WT cells while it does not have any effect in ABCA1 KO cells. Similar results are obtained on miR-143/145, which positively regulate myocardin.

Conclusions. $\mathrm{HDL}_{3}$ modulates the miR143/145-myocardin-KLF4 axis and prevents the cholesterol-induced phenotypic changes in SMC, but only in the presence of a functional ABCA1. 